\title{
INTERACTION OF AROMATIC CYTOKININS WITH HUMAN LIVER MICROSOMAL CYTOCHROMES P450
}

\author{
Eva Anzenbacherováa ${ }^{a}$ Jakub Janalík ${ }^{\mathrm{a}, \mathrm{b}}$, Igor Popa ${ }^{\mathrm{b}}$, Miroslav Strnad ${ }^{\mathrm{b}}$, Pavel Anzenbacher ${ }^{\mathrm{c}}$ \\ a Institute of Medical Chemistry and Biochemistry, Faculty of Medicine, Palacky University, Hnèvotínská 3, 77515 Olomouc, \\ Czech Republic \\ ${ }^{b}$ Laboratory of Growth Regulators, Palacky University and Institute of Experimental Botany, Academy of Sciences Joint \\ Laboratory, Šlechtiteli 11, 78371 Olomouc, Czech Republic \\ c Institute of Pharmacology, Faculty of Medicine, Palacky University, Hnèvotínská 3, 77515 Olomouc, Czech Republic \\ e-mail:anzeneva@tunw.upol.cz
}

Received: June 10, 2005; Accepted: September 25, 2005

Key words: Cytokinins/Cyclin dependent kinase inhibitor/Cytochrome P450/CYP/Human microsomes

Aromatic cytokinins (ortho-topolin riboside, 6-benzylaminopurine riboside and 6-(2-hydroxy-3-methoxybenzyla mino)purine riboside) were tested for their possible interaction with human liver microsomal cytochromes P450 by absorption difference spectroscopy. All three compounds were shown to bind to the CYP enzymes producing a high to low spin shift of the heme iron yielding a Soret absorption band shift to approximately $425 \mathrm{~nm}$. As this type of spectral change means that the substance is able to bind directly to the heme iron, the results obtained open the possibility of an interaction of these compounds with metabolism of other drugs or, in general, with other substrates of cytochromes P450.

\section{INTRODUCTION}

Cytokinins are low molecular weight substances $\left(\mathrm{N}^{6}-\right.$ substituted adenine derivatives) found in plants. Their most important role is to promote cell division and to stimulate morphogenesis ${ }^{1}$. On the contrary, certain cytokinin ribosides cause cell cycle arrest in the animal cell. There are also synthetic cytokinin analogues, acting as inhibitors of cyclin dependent kinases ${ }^{2}$ which are intensively studied as potential antineoplastic drugs ${ }^{3}$. Information on the interaction of any new drug with the main enzymes of drug metabolism (in this case the cytochromes $\mathrm{P} 450$ ) is essential to understanding the mechanism of drug metabolism ${ }^{4}$. Consequently we have performed a pilot study of the interaction of a new class of cytokinin derivatives with cytochromes P450 (CYP).

CYP enzymes involved in metabolism of foreign compounds (xenobiotics like drugs, food additives, and environmental pollutants) are localized mostly in the endoplasmatic reticulum of hepatocytes; however, they are ubiquitous, being present also in other tissues such as intestine, lung, kidney or brain ${ }^{4}$. There are about eight CYPs responsible for over $95 \%$ of known cases of drug-dependent cytochrome P450 metabolism: CYP3A4, CYP2D6, CYP2C9, CYP2E1, CYP2C19, CYP1A2, CYP2A6 and CYP2B $6{ }^{5}$. In this study, we investigated whether the compounds studied (three cytokinin derivatives with the $\mathrm{N}^{6}$-amino group of adenine substituted with aromatic substituents, hence conventionally named "aromatic" cytokinins) are able to interact with CYP enzymes present in human liver microsomes.

\section{MATERIAL AND METHODS}

\section{Material}

Laboratory chemicals were purchased from Sigma Aldrich CZ (Prague) and were of reagent grade purity. Three cytokinins, ortho-topolin riboside (oTR), 6-benzylaminopurine riboside (BAPR) and 6-(2-hydroxy-3-metho xybenzylamino)purine riboside (HMBAPR) were either also purchased from Sigma (BAPR) or synthesized in our laboratory (Laboratory of Growth Regulators, oTR and HMBAPR). Structures of these compounds are shown in Fig. 1. The microsomal fraction of human liver homogenate was purchased from Advancell (Barcelona).

\section{Methods}

Difference spectra of interactions of cytokinins with microsomal CYP enzymes were followed according to established procedure ${ }^{6}$. The compounds to be tested were dissolved in an appropriate solvent ( $70 \%$ ethanol). This
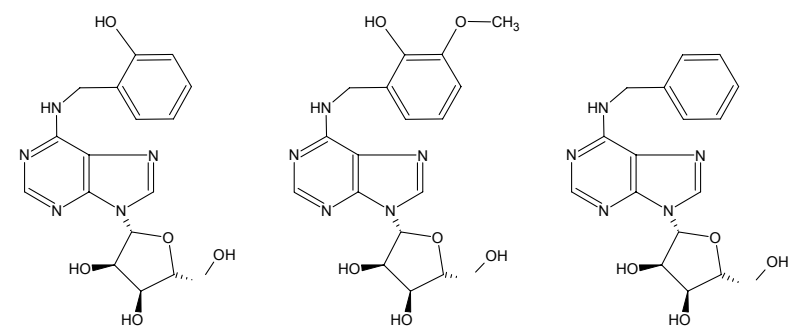

Fig. 1. Structures of three aromatic cytokinins (OTR, HMBAPR and BAPR). 
stock was further diluted in the respective media used in each assay. The final concentration of ethanol in the media did not exceed $1 \%$, and therefore did not interfere with native CYPs used. An adequate amount of solvent was added to the reference cuvette. Spectra were recorded on a Specord UV VIS M40 spectrophotometer (Carl Zeiss, Jena). The spectral changes (absorbance difference at the peak maximum) generally follow a rectangular hyperbola ${ }^{6}$. The respective values were plotted to obtain the value of spectroscopic binding constant $K_{\mathrm{s}}$ and of the limiting value of the spectral change ( $A_{\max }$, with meaning of the CYP binding capacity of the enzymes) using the Sigma Plot v. 8.0 graphing and statistical software (SPSS, Chicago, IL).

\section{RESULTS AND DISCUSSION}

\section{Spectroscopic study of interaction of cytokinins with microsomal CYP}

Interaction of all three substances with human microsomal fraction resulted in a characteristic difference spectrum with maximum at about $425 \mathrm{~nm}$ and minimum at $400 \mathrm{~nm}$. This type of spectral change was formerly named as a Type II difference spectrum. A typical spectrum obtained with oTR is displayed in Fig. 2.

In such a case, the course of the spectra is given by a spin shift of the heme iron atom from the high to the low spin state. As the low spin state of the heme iron is manifested by a Soret absorption maximum at about $420 \mathrm{~nm}$ and the high spin state of the heme iron atom is known to be reflected by the position of the Soret band at about $390 \mathrm{~nm}$, a high to low spin shift is expressed in the difference absorption spectrum (in a sample with tested compound + microsomal fraction in the sample cuvette and with the microsomal fraction alone in the reference cuvette) exactly as shown in the Fig. 2. Low spin of the heme iron is, however, typical of the hexacoordinated heme iron with substrate (or, generally, of an interacting compound) bound through its donor atoms to the heme iron. In fact, all three cytokinins possess functional groups or atoms which make this kind of interaction possible, namely, the nitrogen atoms of the adenine skeleton or amino group as well as the hydroxyl groups of oTR and HMBAPR.

\section{Analysis of binding of cytokinins to microsomal CYP}

The curve expressing the change of absorbance in the Soret absorption band region with increasing concentration of substrate should be a rectangular hyperbola ${ }^{6}$. The

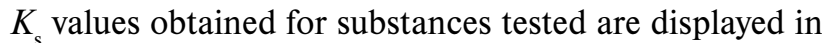
Table 1. For illustration, the experimental data are shown in Fig. 3.

Interestingly, the $K_{\mathrm{s}}$ values reflect the structure of the respective molecules: BAPR is the most nonpolar cytokinin with an unsubstituted aromatic ring; HMBAPR possesses two substituents of the aromatic ring: a hydroxyl and a methoxy group. As cytochromes P450 tend to bind

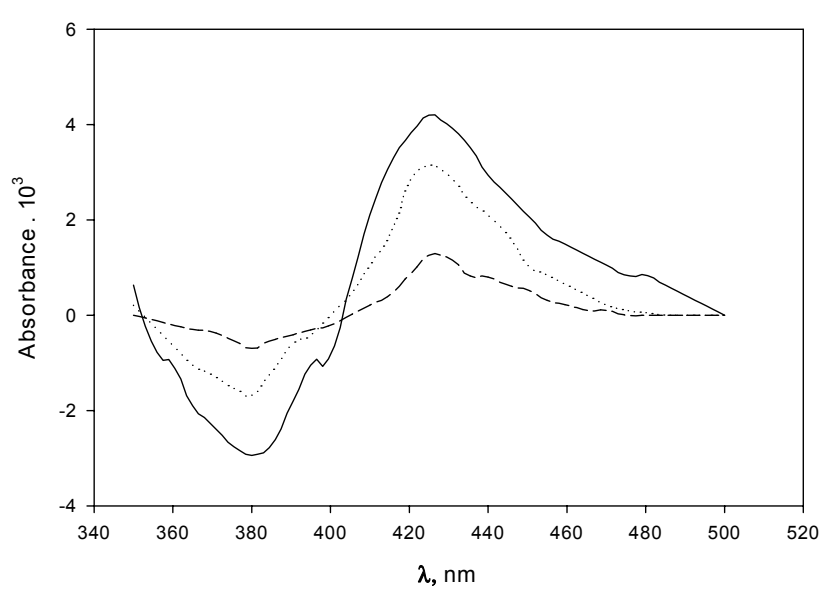

Fig. 2. Difference spectra of interaction of human liver microsomal cytochrome P450 with OTR.

CYP concentration $1.41 \mu \mathrm{M}$, OTR $10 \mu \mathrm{M}$ (dashed), $16 \mu \mathrm{M}$ (dotted), $26 \mu \mathrm{M}$ (full line).

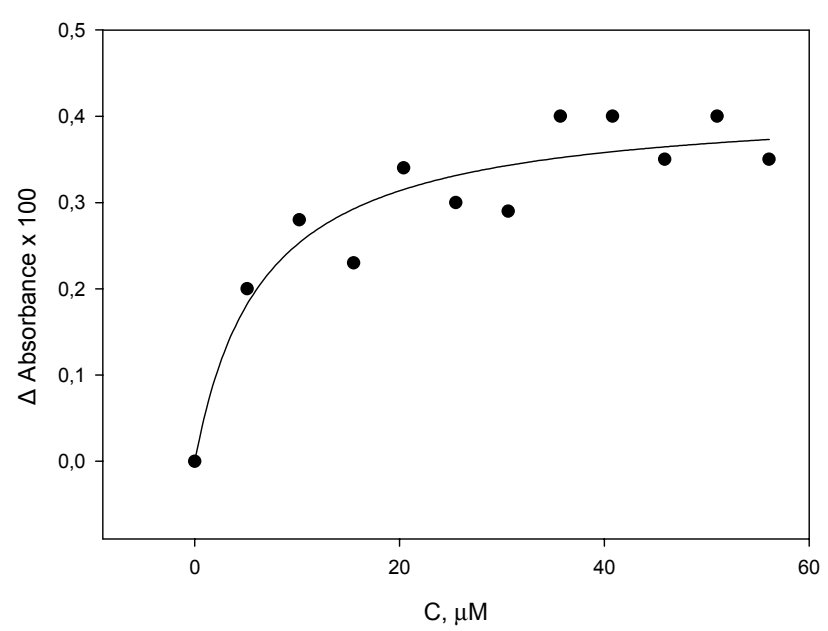

Fig. 3. Binding of BAPR to microsomal CYP followed by difference spectroscopy.

Table 1. Spectroscopic binding constants (Ks) and limiting absorbance values (Amax) for interaction of cytokinins with human liver microsomal CYP

\begin{tabular}{|c|c|c|c|}
\hline & BAPR & oTR & HMBAPR \\
\hline $\begin{array}{c}K_{\mathrm{s}} \\
(\mu \mathrm{M})\end{array}$ & $6.1 \pm 2.3$ & $7.23 \pm 2.0$ & $11.6 \pm 2,4$ \\
\hline $\mathrm{A}_{\max }$ & $0.0041 \pm 0.003$ & $0.0040 \pm 0.0003$ & $0.016 \pm 0.0001$ \\
\hline
\end{tabular}


the nonpolar molecules and convert them to their more polar derivatives, the result seems to be logical.

The results presented here, however, strongly indicate the possibility of direct interaction of these cytokinins with cytochromes P450. As the liver microsomal cytochromes are known to metabolize a variety of substrates including drugs, this interaction might be important for drug-drug interactions. In other words, a detailed study aimed at individual CYP forms is needed to answer the question whether the cytokinins studied may interfere with metabolism of drugs and other substrates of CYP enzymes.

\section{ACKNOWLEDGEMENT}

Financial support through the MSM 6198959216 project of the Czech Ministry of Education, Youth and Sports is gratefully acknowledged.

\section{REFERENCES}

1. Davies PJ. Plant Hormones: Physiology, Biochemistry and Molecular Biology. Dordrecht: Kluwer, 1995

2. Hajduch M, Kolar Z, Novotny R, Hanus J, Mihal V, Hlobilkova A, Noskova V, Strnad M. (1997) Induction of apoptosis and regression of spontaneous dog melanoma following in vivo application of synthetic cyclin-dependent kinase inhibitor olomoucine. Anticancer Drugs 8, 1007-13.

3. Veselý J, J, Havlíček L, Strnad M, Blow JJ, Donella-Deana A, Pinna L, Letham DS, Kato J, Detivaud L, Leclerc S, Meijer L. (1994) Inhibition of cyclin-dependent kinases by purine analogues. Eur J Biochem 224, 771-86.

4. Anzenbacher P, Anzenbacherová E. (2001) Cytochromes P450 and metabolism of xenobiotics. CMLS, Cell Mol Life Sci 58, 737-47.

5. Guengerich FP, Human cytochromes P450. In: Ortiz de Montellano PR, editor. Cytochrome P450, structure, mechanism and biochemistry ( $3^{\text {rd }}$ Ed.). New York, Kluwer/Plenum Press, 2005. p. $377-$ 530 .

6. Schenkman JB, Jansson I. Spectral analyses of cytochromes P450. In: Phillips IR, Shephard EA, editors. Cytochrome P450 Protocols (Meth Mol Biol 107). Totowa: Humana Press, 1998. p. 25-33. 